\title{
Successive Interference Cancellation for Multiuser Asynchronous DS/CDMA Detectors in Multipath Fading Links
}

\author{
Andrew L. C. Hui and Khaled Ben Letaief, Senior Member, IEEE
}

\begin{abstract}
In recent years, there has been an increasing interest in the use of code-division multiple access (CDMA) in cellular mobile and wireless personal communications. The choice of such multiaccess technique is attractive because of its potential capacity increases and other technical factors such as privacy and multipath rejection capabilities. However, it is well known that the performance of CDMA can be significantly degraded due to cochannel interference (CI) and the near-far effects. In this paper we consider the performance of direct-sequence (DS)based CDMA over fading channels that are modeled as slowly varying Rayleigh-fading discrete multipath channels. Specifically, we propose and analyze an adaptive multistage interference cancellation strategy for the demodulation of asynchronous DS spread-spectrum multiple-access signals. Numerical results will show that the proposed multistage detector, which alleviates the detrimental effects of the near-far problem, can significantly improve the system performance.
\end{abstract}

Index Terms - Cochannel interference cancellation, code-division multiple access, multiuser detection, wireless communications.

\section{INTRODUCTION}

$\mathbf{R}$ ECENTLY, direct-sequence code-division multiple access (DS/CDMA) has become very popular in various applications such as optical and wireless communications [1], [2]. However, it is well known that the conventional CDMA receiver does suffer from serious problems. For example, as the number of users increases it can become multipleaccess interference limited. Another major disadvantage is the near-far problem, which refers to the phenomenon of high-power interferers completely destroying communications from lower power transmitters. These disadvantages raise the question of whether the problems described above are inherent shortcomings of DS/CDMA and whether significant performance improvement might be possible through the use of other receivers. In recent years, there has been a lot of interest in multiuser detection in which these questions are addressed. This interest was motivated by the work of

Paper approved by R. Kohno, the Editor for Spread Spectrum Theory and Applications of the IEEE Communications Society. Manuscript received September 13, 1996; revised November 13, 1996. This work was supported in part by the Hong Kong Grant Council under Grant HKUST 562/94E.

The authors are with the Electrical and Electronic Engineering Department, The Hong Kong University of Science \& Technology, Clear Water Bay, Kowloon, Hong Kong (e-mail: eekhaled@ee.ust.hk).

Publisher Item Identifier S 0090-6778(98)02126-6.
Verdú in [3] where it has been shown that the optimum multiuser CDMA detector is near-far resistant and that significant performance can be gained by optimum, as opposed to conventional, demodulation. Unfortunately, the optimum multiuser detector has three disadvantages - it requires centralized implementation, it is quite complex, and it requires knowledge of the received energies of the various users. Specifically, it is a maximum-likelihood detector which consists of a bank of conventional single-user matched filters followed by a Viterbi algorithm whose complexity grows exponentially as the number of users increases. ${ }^{1}$ Further, it has been shown that the multiuser optimum decision problem is NP-hard. To reduce the complexity of this optimum detector various suboptimum multiuser detectors have been developed in the past several years [4]-[7]. These detectors vary in complexity and have been mainly studied over the additive white Gaussian noise (AWGN) channel. Interested readers are referred to [8]-[10] and references therein for further details.

In this paper we propose a multiuser detection methodology for DS/CDMA communications over multipath Rayleighfading links. The proposed method consists of a serial cochannel interference (CI) cancellation scheme that is based on the one described in [8]. Specifically, the proposed multistage detector aims to improve the system performance by regenerating estimates of the interfering signals, and then subtract those reconstructed interference signals from the input of the desired receiver. This process is performed in a cascaded fashion in such a way that more and more of the "strongest" interfering signals are canceled. Another key objective of this paper is to present a performance analysis that is applicable to our proposed interference cancellation method as well as the one proposed in [8].

This paper is organized as follows. In Section II we will describe the DS/CDMA system along with the multipath channel. Section III presents the proposed CI cancellation methodology. In Section IV an error performance analysis of the CI cancellation method is developed. Section V presents an analytical approach for optimizing the performance of the proposed detector. Section VI presents some sample numerical results and Section VII is the concluding section.

\footnotetext{
${ }^{1}$ The complexity is actually $O\left(2^{K}\right)$ where $K$ is the total number of users.
} 


\section{SYSTEM DESCRIPTION}

Consider an asynchronous DS/CDMA system [11], [12], [14] which consists of $K$ users, and let $b_{k}(t)=$ $\sum_{j=-\infty}^{\infty} b_{j}^{(k)} p_{t}(j T,(j+1) T)$ denote the binary data signal, and $a_{k}(t)=\sum_{j=-\infty}^{\infty} \quad a_{j}^{(k)} p_{t}\left(j T_{c},(j+1) T_{c}\right)$ denote the signature sequence signal with $p_{t}\left(t_{1}, t_{2}\right)$ being a unit rectangular pulse on $\left[t_{1}, t_{2}\right), b_{j}^{(k)}= \pm 1$ with equal probabilities, and the $k$ th user's code sequence $a_{j}^{(k)} \in\{-1,1\}$ with $a_{j}^{(k)}=a_{j+N}^{(k)}$ for all $j$ and $k$ with the integer $N \equiv T / T_{c}$. Next, consider the transmission over a multipath Rayleighfading channel having a slow fading rate compared to the bit rate, so that the channel random parameters do not change significantly over several bit intervals. Specifically, throughout it is assumed that the complex low-pass equivalent impulse response of the channel is given by

$$
h_{k}(t)=\sum_{\ell=1}^{L_{k}} \beta_{\ell}^{(k)} \delta\left(t-\tau_{\ell}^{(k)}\right) e^{\imath \gamma_{\ell}^{(k)}}
$$

where $\beta_{\ell}^{(k)}, \tau_{\ell}^{(k)}$, and $\gamma_{\ell}^{(k)}$ are the $\ell$ th path gain, delay, and phase for the $k$ th user $\imath \triangleq \sqrt{-1}$, and $L_{k}$ is the number of paths which can be either deterministic or random. Let $\Delta$ denote the maximum delay spread, which is assumed to be small compared to the symbol duration $T$ so that intersymbol interference (ISI) would be small. Then, the path delay $\tau_{\ell}^{(k)}$ will be assumed to be uniformly distributed over $\left[T_{c}, \Delta\right]$. Likewise, the path gains $\beta_{\ell}^{(k)}$ will be assumed to be independent Rayleigh random variables with the average path power $\mathrm{E}\left[\left(\beta_{\ell}^{(k)}\right)^{2}\right] \equiv 2 \rho$ for all $k$ and $\ell$ and $\mathrm{E}[\cdot]$ is the expectation operator.

Now, let $r(t)$ denote the received signal, then it can be easily shown that

$$
\begin{array}{r}
r(t)=\sum_{k=1}^{K} \sum_{\ell=1}^{L_{k}} \sqrt{2 P_{k}} \beta_{\ell}^{(k)} b_{k}\left(t-t_{\ell}^{(k)}\right) a_{k}\left(t-t_{\ell}^{(k)}\right) \\
\cdot \cos \left(\omega_{c} t+\phi_{\ell}^{(k)}\right)+n(t)
\end{array}
$$

where $n(t)$ is a white Gaussian noise with double-sided power spectral density $N_{0} / 2, P_{k}=E_{k} / T$ is the $k$ th user signal power, $\omega_{c}$ is the carrier frequency, and $\phi_{\ell}^{(k)} \equiv \gamma_{\ell}^{(k)}-\omega_{c} \tau_{\ell}^{(k)}-$ $\omega_{c} T_{k}$ is the $\ell$ th path phase of the $k$ th user received signal with $T_{k}$ denoting the $k$ th user asynchronous transmission delay. Throughout, we will assume that $\phi_{\ell}^{(k)}$ is an independent random variable uniformly distributed over $[0,2 \pi]$ for each $\ell$ and $k$. In addition, we will assume the overall delay $t_{\ell}^{(k)}=\tau_{\ell}^{(k)}+T_{k}$ to be an independent random variable that is uniformly distributed over $[0, T]$.

\section{The CI Cancellation Method}

Without loss of generality, we assume that the desired user is the $i$ th user. Further, we assume that the desired receiver can coherently recover the carrier phase and delay lock to the first arriving path of the desired user's signal. Therefore, if we let $\mathcal{X}_{i}$ denote the output of the correlator receiver matched to the first path of the $i$ th user at $t=n T+t_{1}^{(i)}$, then

$$
\begin{aligned}
\mathcal{X}_{i}= & \sqrt{\frac{P_{i}}{2}} b_{n}^{(i)} T \beta_{1}^{(i)} \sqrt{\frac{P_{i}}{2}} b_{n}^{(i)} T \beta_{1}^{(i)}+\sqrt{\frac{P_{i}}{2}} \sum_{\ell=2}^{L_{i}} x_{\ell}^{(i)} \\
& +\sum_{k=1 k \neq i}^{K} \sum_{\ell=1}^{L_{k}} \sqrt{\frac{P_{k}}{2}} x_{\ell}^{(k)}+\eta_{i}
\end{aligned}
$$

where

$$
\begin{aligned}
\eta_{i}= & \int_{(n-1) T+t_{1}^{(i)}}^{n T+t_{1}^{(i)}} n(t) a_{i}\left(t-t_{1}^{(i)}\right) \cos \left(\omega_{c} t+\phi_{1}^{(i)}\right) d t \\
x_{\ell}^{(k)} \triangleq & \beta_{\ell}^{(k)} \cos \left(\phi_{\ell}^{(k)}-\phi_{1}^{(i)}\right) \\
& \cdot\left[b_{n-m-1}^{(k)} R^{(k i)}\left(t_{\ell}^{(k)}-t_{1}^{(i)}-m T\right)\right. \\
& \left.+b_{n-m}^{(k)} \hat{R}^{(k i)}\left(t_{\ell}^{(k)}-t_{1}^{(i)}-m T\right)\right]
\end{aligned}
$$

and $\left.m=\mid t_{\ell}^{(k)}-t_{1}^{(i)} / T\right\rfloor$. Likewise, $R^{(k i)}(\tau)=\int_{0}^{\tau} a_{k}(t-$ $\tau) a_{i}(t) d t$ and $\hat{R}^{(k i)}(\tau)=\int_{\tau}^{T} a_{k}(t-\tau) a_{i}(t) d t$ for $0 \leq \tau \leq T$, $\ell=1,2, \cdots, L_{k}$, and $k=1,2, \cdots, K$ are the continuous-time partial correlation functions. Now let $D_{i} \triangleq \beta_{1}^{(i)} \sqrt{P_{i} / 2} T b_{n}^{(i)}$ denote the correlator's output of the $i$ th desired user signal. Also let

$$
S_{i} \triangleq \sqrt{\frac{P_{i}}{2}} \sum_{\ell=2}^{L_{i}} x_{\ell}^{(i)} \quad C_{i} \triangleq \sum_{k=1 k \neq i}^{K} \sum_{\ell=1}^{L_{k}} \sqrt{P_{k} / 2} x_{\ell}^{(k)}
$$

denote the output of the desired user "self" interference and the desired user total multiuser interference, respectively. Then, the lump sum of $S_{i}$ and $C_{i}$ will be referred to as the CI for the desired user.

Next, note that to combat the CI, one must minimize the sum of $S_{i}$ and $C_{i}$. The basic idea behind the proposed method is to reproduce the CI terms $S_{i}$ and $C_{i}$ and then subtract the replicas of $S_{i}$ and $C_{i}$ from the $i$ th user received signal. This process is performed successively. Specifically, we first find initial estimates of the CI terms (stage 0). We will then proceed with $V$ stages of processing the decision statistics of the desired user. At the $v$ th stage, we reconstruct the CI terms using estimates of the channel parameters and the unknown transmitted symbols and then subtract the regenerated CI for the desired user received signal to obtain a new decision statistic. Ideally, if we can correctly estimate $S_{i}$ and $C_{i}$ at say stage $v$, then the decision statistic at the next stage will be simply equal to $D_{i}+\eta_{i}$. Consequently, significant improvement in the system performance would result. On the other hand, if the data estimation for say user $k$ is incorrect at say stage $v$, then a negative version of the interference caused by this user is created, thereby resulting in a new CI term (due to user $k$ ) at the $v+1$ stage that is twice the CI term of user $k$ at stage $v$. It is therefore clear that one must have good data estimates (and, hence, good estimates of the CI terms) in order for this iterative scheme to work well.

In [8], a successive CI cancellation methodology as described above has been proposed. However, Yoon et al. proposed that the data estimation be obtained using a hard decision upon the decision statistic at every stage. Since we are considering wireless channels that are characterized by 


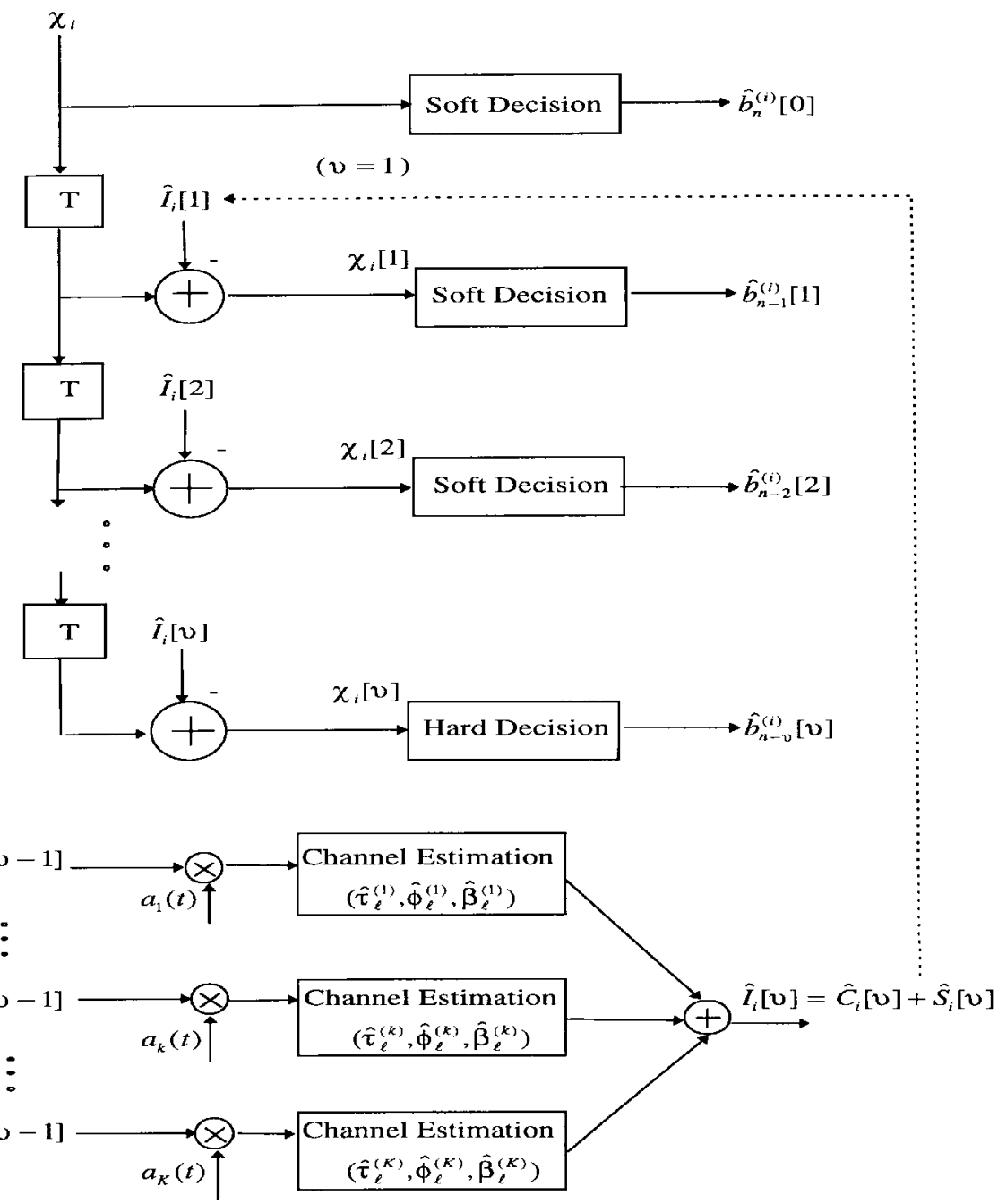

Fig. 1. DS/CDMA receiver with multistage cochannel interference cancellation.

multipath fading links along with imperfect power control, one would expect the occurence of incorrect data estimates (especially at the initial stage) to be relatively high, ${ }^{2}$ thus possibly resulting in an increase in CI. To solve this problem and in order to have good data estimates, here we propose a detection scheme which tends to decode the strongest signals and then cancel their CI terms from the received signals as shown in Fig. 1. Specifically, to make the data estimation more accurate for the CI cancellation scheme, we will use a soft decision upon the correlator output at the various stages. More precisely, our data estimation is performed as follows. During the $v$ th stage $(v=1,2, \cdots, V)$ we let $\hat{S}_{i}[v]$ and $\hat{C}_{i}[v]$ denote the replicas of $S_{i}$ and $C_{i}$, respectively. Then, by removing these terms from $\mathcal{X}_{i}$ and performing the CI on the $(n-v)$ th bit of the $i$ th user, we obtain the decision statistic at the $v$ th stage

$$
\begin{aligned}
\mathcal{X}_{i}[v] & =\mathcal{X}_{i}-\hat{S}_{i}[v]-\hat{C}_{i}[v] \\
& \equiv \beta_{1}^{(i)} \sqrt{\frac{P_{i}}{2}} b_{n-v}^{(i)} T+\tilde{S}_{i}[v]+\tilde{C}_{i}[v]+\eta_{i}
\end{aligned}
$$

\footnotetext{
${ }^{2}$ In fact, this is precisely the reason why a good CI interference scheme is desirable.
}

where $\tilde{S}_{i}[v]=S_{i}-\hat{S}_{i}[v]$ and $\tilde{C}_{i}[v]=C_{i}-\hat{C}_{i}[v]$. Therefore, it follows that

$$
\begin{aligned}
\mathcal{X}_{i}[v]= & \eta_{i}+\beta_{1}^{(i)} \sqrt{\frac{P_{i}}{2}} b_{n-v}^{(i)} T+\sum_{\ell=2}^{L_{i}} \sqrt{\frac{P_{i}}{2}} \beta_{\ell}^{(i)} \cos \left(\phi_{\ell}^{(i)}-\phi_{1}^{(i)}\right) \\
& \times\left[\tilde{b}_{n-v-1}^{(i)}[v-1] R^{(i i)}\left(t_{\ell}^{(i)}-t_{1}^{(i)}\right)\right. \\
& \left.+\tilde{b}_{n-v}^{(i)}[v-1] \hat{R}^{(i i)}\left(t_{\ell}^{(i)}-t_{1}^{(i)}\right)\right] \\
& +\sum_{k=1 k \neq i}^{K} \sum_{\ell=1}^{L_{k}} \sqrt{\frac{P_{k}}{2}} \beta_{\ell}^{(k)} \cos \left(\phi_{\ell}^{(k)}-\phi_{1}^{(i)}\right) \\
& \times\left[\tilde{b}_{n-v-1-m}^{(k)}[v-1] R^{(k i)}\left(t_{\ell}^{(k)}-t_{1}^{(i)}-m T\right)\right. \\
& \left.+\tilde{b}_{n-v-m}^{(k)}[v-1] \hat{R}^{(k i)}\left(t_{\ell}^{(k)}-t_{1}^{(i)}-m T\right)\right]
\end{aligned}
$$

where $\tilde{b}_{j}^{(k)}[v-1] \triangleq b_{j}^{(k)}-\hat{b}_{j}^{(k)}[v-1], m$ is the number of shifted bits relative to the first path of user $i$ and $\hat{b}_{j}^{(k)}[v-1]$ is the data estimate at the $(v-1)$ th stage. The soft decision CI method then proceeds as follows. If $v \neq V$, then the bit decision for user $i$ 's $(n-v)$ th bit is

$$
\hat{b}_{n-v}^{(i)}[v]= \begin{cases}-1, & \mathcal{X}_{i}[v] \leq-\mathcal{T}_{v}^{(i)} \\ \text { no decision, } & \left|\mathcal{X}_{i}[v]\right|<\mathcal{T}_{v}^{(i)} \\ 1, & \text { else. }\end{cases}
$$


Otherwise, we have $\hat{b}_{n-v}^{(i)}[v]=\operatorname{sgn}\left(\mathcal{X}_{i}[v]\right)$ where $\mathcal{T}_{v}^{(i)}>0$ is a given constant threshold for the $i$ th user $(v=0,1, \cdots, V-1)$ and $\operatorname{sgn}(x)=1$ if $x>0$. Else, $\operatorname{sgn}(x)=0$. In other words, we perform data estimation if and only if the decision statistic is greater than or smaller than $\mathcal{T}_{v}^{(i)}$. Otherwise, no data estimation is made for user $i$. In particular, if the $v$ th stage is actually the final stage (i.e., $V=v$ ), then a hard decision is used instead. Clearly, the proper choice of all $\mathcal{T}_{v}^{(i)}$ is important for the successful implementation of the proposed cancellation method. In particular, we note that the proposed method reduces to the one presented in [8] when the thresholds $\mathcal{T}_{v}^{(k)}$ are equal to zero for all $v$ and $k$.

\section{Performance Analysis}

The main objective of this section is to present a closed form expression for the system's average bit-error rate (BER). We begin by deriving the system's BER in the initial stage.

\section{A. Initial Stage}

Let $\mathcal{X}_{i}, S_{i}, C_{i}$, and $\eta_{i}$ be defined as above. Then, it can be easily shown that $\operatorname{var}\left[\eta_{i}\right]=N_{o} T / 4$

$$
\begin{aligned}
\operatorname{var}\left[S_{i}\right]= & \frac{P_{i}}{4\left(\Delta-T_{c}\right)}\left(\overline{L_{i}}-1\right) \mathrm{E}\left[\left(\beta_{\ell}^{(i)}\right)^{2}\right] \\
& \cdot \int_{T_{c}}^{\Delta}\left(R^{i i}(\tau)\right)^{2}+\left(\hat{R}^{i}(\tau)\right)^{2} d \tau \\
\operatorname{var}\left[C_{i}\right]= & \sum_{k=1}^{K} \frac{P_{k} T^{2}}{12 N^{3}}\left(\overline{L_{k}}\right) \mathrm{E}\left[\left(\beta_{\ell}^{(k)}\right)^{2}\right] r_{k i}
\end{aligned}
$$

where

$$
\begin{aligned}
r_{k i}= & \sum_{j=1}^{N-1}\left[\left(C^{k i}(j-N)\right)^{2}+C^{k i}(j-N) C^{k i}(j-N+1)\right. \\
& +\left(C^{k i}(j-N+1)\right)^{2}+\left(C^{k i}(j)\right)^{2} \\
& \left.+C^{k i}(j) C^{k i}(j+1)+\left(C^{k i}(j+1)\right)^{2}\right]
\end{aligned}
$$

and $C^{k i}(\cdot)$ is the signature sequence aperiodic cross correlation function [12], [13].

The BER can now be obtained by assuming the CI terms to be Gaussian distributed. This may not be necessarily correct, but such an approximation has been shown to be relatively good [8], [14]. Now recall that $D_{i}=\beta_{1}^{(i)} \sqrt{\left(P_{i} / 2\right)} T b_{n}^{(i)}$; then, for a given $\beta=\beta_{1}^{(i)}$, it follows that the conditional signalto-noise ratio (SNR) for the $i$ th user's correlator output is $S / N_{i}=\beta^{2}\left(P_{i} / 2\right) T^{2}\left(\operatorname{var}\left[C_{i}\right]+\operatorname{var}\left[S_{i}\right]+\operatorname{var}\left[\eta_{i}\right]\right)$ or

$$
\begin{aligned}
\left(S / N_{i}\right)^{-1} \equiv & \frac{\left(\overline{L_{i}}-1\right) \mathrm{E}\left[\left(\beta_{\ell}^{(i)}\right)^{2}\right] A_{i}}{2 \beta^{2} T^{2}} \\
& +\frac{\sum_{k=1}^{K} \frac{P_{k}}{P_{i}} \overline{L_{k}} \mathrm{E}\left[\left(\beta_{\ell}^{(k)}\right)^{2}\right] r_{k i}}{6 N^{3} \beta^{2}}+\frac{N_{o}}{2 \beta^{2} E_{i}} .
\end{aligned}
$$

As a result

$$
\begin{aligned}
P_{e}(\beta) & \triangleq \operatorname{Pr}\left(\text { Error at the initial stage } \mid \beta_{1}^{(i)}=\beta\right) \\
& =Q\left[\sqrt{S / N_{i}}\right]
\end{aligned}
$$

where $Q[x]=(1 / \sqrt{2 \pi}) \int_{x}^{\infty} \exp \left(-\left(t^{2} / 2\right)\right) d t$. The error probability for the initial stage can now be obtained by integrating $P_{e}(\beta)$ given by (12) with respect to the density of $\beta$. As a result, we get

$$
P_{e}[0]=\frac{1}{2}\left(1-\sqrt{\frac{\bar{\gamma}_{i}}{1+\bar{\gamma}_{i}}}\right)
$$

where $\bar{\gamma}_{i} \triangleq \frac{1}{2} \mathrm{E}\left[S / N_{i}\right]$ and the expectation is with respect to $\beta$.

\section{B. Error Rate in the First Stage}

We begin by deriving an expression for $P_{e}^{(i)}$ and $P_{t}^{(i)}$ where $P_{e}^{(i)}$ and $P_{t}^{(i)}$ are the probability of making an error and the probability that no decision is made at the initial stage for the $i$ th user, respectively. Due to the symmetry of the problem, one can without loss of generality set $b_{n}^{(i)}=-1$. Hence, $P_{e}^{(i)} \equiv$ $P\left(\mathcal{X}_{i}>\mathcal{T}_{0}^{(i)} \mid b_{n}^{(i)}=-1\right)$ and $P_{t}^{(i)} \equiv P\left(\left|\mathcal{X}_{i}\right|<\mathcal{T}_{0}^{(i)} \mid b_{n}^{(i)}=\right.$ $-1)$. Next, let

$$
\mathrm{SNR}_{i} \triangleq \frac{\frac{P_{i}}{2} T^{2}}{\operatorname{var}\left[\eta_{i}\right]+\operatorname{var}\left[S_{i}\right]+\operatorname{var}\left[C_{i}\right]}
$$

and

$$
\xi_{0}^{(i)} \triangleq \frac{\mathcal{T}_{0}^{(i)}}{\sqrt{\operatorname{var}\left[\eta_{i}\right]+\operatorname{var}\left[S_{i}\right]+\operatorname{var}\left[C_{i}\right]}}
$$

Likewise, let $f_{\beta_{1}^{(i)}}(\beta)$ be the density function of $\beta_{1}^{(i)}$. Then, it can be easily shown that

$$
P_{e}^{(i)}=\int_{0}^{\infty} Q\left[\xi_{0}^{(i)}+\beta \sqrt{\mathrm{SNR}_{i}}\right] f_{\beta_{1}^{(i)}}(\beta) d \beta
$$

and

$$
\begin{aligned}
P_{t}^{(i)}= & \int_{\xi_{0}^{(i)} / \sqrt{\mathrm{SNR}_{i}}}^{\infty}\left(Q\left[\beta \sqrt{\mathrm{SNR}_{i}}-\xi_{0}^{(i)}\right]\right. \\
& \left.-Q\left[\xi_{0}^{(i)}+\beta \sqrt{\mathrm{SNR}_{i}}\right]\right) f_{\beta_{1}^{(i)}}(\beta) d \beta \\
& +\int_{0}^{\xi_{0}^{(i)} / \sqrt{\mathrm{SNR}_{i}}}\left(1-Q\left[\xi_{0}^{(i)}-\beta \sqrt{\mathrm{SNR}_{i}}\right]\right. \\
& \left.-Q\left[\xi_{0}^{(i)}+\beta \sqrt{\mathrm{SNR}_{i}}\right]\right) f_{\beta_{1}^{(i)}}(\beta) d \beta
\end{aligned}
$$

Now note that only the CI terms are different in the decision statistic of the single-stage CI cancellation process. In particular, we have

$$
\operatorname{var}\left[\widetilde{S}_{i}[1]\right]=\mathrm{E}\left[\left(\tilde{b}_{n}^{(i)}[0]\right)^{2}\right] \operatorname{var}\left[S_{i}\right]
$$

and

$$
\operatorname{var}\left[\tilde{C}_{i}[1]\right]=\sum_{k=1 k \neq i}^{K} \mathrm{E}\left[\left(\tilde{b}_{n}^{(k)}[0]\right)^{2}\right] \operatorname{var}\left[C_{i}^{(k i)}\right]
$$


where

$$
\begin{aligned}
\operatorname{var}\left[C_{i}^{(k i)}\right]= & \frac{P_{k} T^{2}}{12 N^{3}}\left(\overline{L_{k}}\right) \mathrm{E}\left[\left(\beta_{\ell}^{(k)}\right)^{2}\right] r_{k i} \\
\mathrm{E}\left[\left(\tilde{b}_{n}^{(k)}[0]\right)^{2}\right]= & 1+2 Q\left[\xi_{0}^{(k)}\right]-\sqrt{\frac{\bar{\gamma}_{k}}{\bar{\gamma}_{k}+1}} \exp \\
& \cdot\left(-\frac{\left(\xi_{0}^{(k)}\right)^{2}}{2\left(\bar{\gamma}_{k}+1\right)}\right)\left[1+2 Q\left[\sqrt{\frac{\left(\xi_{0}^{(k)}\right)^{2} \bar{\gamma}_{k}}{\bar{\gamma}_{k}+1}}\right]\right]
\end{aligned}
$$

and $r_{k i}$ is defined in (10). As a result, it follows that the BER for the first stage of the CI cancellation method is given by

$$
P_{e}[1]=\frac{1}{2}\left(1-\sqrt{\frac{\bar{\gamma}_{i}[1]}{1+\bar{\gamma}_{i}[1]}}\right)
$$

where $\bar{\gamma}_{i}[1]=\frac{1}{2} \mathrm{E}\left[S / N_{i}[1]\right]$, and

$$
S / N_{i}[1]=\frac{\beta^{2} \frac{P_{i}}{2} T^{2}}{\operatorname{var}\left[\tilde{C}_{i}[1]\right]+\operatorname{var}\left[\tilde{S}_{i}[1]\right]+\operatorname{var}\left[\eta_{i}\right]} .
$$

\section{Error Rate in Successive CI Cancellation Stages}

The extension of the analysis described in the previous section to a $v$-stage detector is straightforward. Indeed, we have

$$
\begin{aligned}
\operatorname{var}\left[\tilde{S}_{i}[v]\right] & =\mathrm{E}\left[\left(\tilde{b}_{n}^{(i)}[v-1]\right)^{2}\right] \operatorname{var}\left[S_{i}\right] \\
\operatorname{var}\left[\tilde{C}_{i}[v]\right] & =\sum_{k=1}^{K} \mathrm{E}\left[\left(\tilde{b}_{n}^{(k)}[v-1]\right)^{2}\right] \operatorname{var}\left[C_{i}^{(k i)}\right]
\end{aligned}
$$

and the second moment of the canceled bit $\mathrm{E}\left[\left(\tilde{b}_{n}^{(k)}[v-1]\right)^{2}\right]$ can be easily shown to be given by

$$
\begin{aligned}
\mathrm{E}\left[\left(\tilde{b}_{n}^{(k)}[v-1]\right)^{2}\right] & 1+2 Q\left[\xi_{v-1}^{(k)}\right]-\sqrt{\frac{\bar{\gamma}_{k}[v-1]}{\bar{\gamma}_{k}[v-1]+1}} \\
\cdot & \exp \left(-\frac{\left(\xi_{v-1}^{(k)}\right)^{2}}{2\left(\bar{\gamma}_{k}[v-1]+1\right)}\right) \\
\cdot & {\left[1+2 Q\left[\sqrt{\frac{\left(\xi_{v-1}^{(k)}\right)^{2} \bar{\gamma}_{k}[v-1]}{\bar{\gamma}_{k}[v-1]+1}}\right]\right] }
\end{aligned}
$$

where

$$
\xi_{v-1}^{(k)}=\frac{\mathcal{T}_{v-1}^{(k)}}{\sqrt{\operatorname{var}\left[\tilde{C}_{k}[v]\right]+\operatorname{var}\left[\tilde{S}_{k}[v]\right]+\operatorname{var}\left[\eta_{k}\right]}} .
$$

Thus, for a given $\beta=\beta_{1}^{(i)}$, it follows that the $v$ th stage SNR of the $i$ th user is

$$
S / N_{i}[v]=\frac{\beta^{2} \frac{P_{i}}{2} T^{2}}{\operatorname{var}\left[\tilde{C}_{i}[v]\right]+\operatorname{var}\left[\widetilde{S}_{i}[v]\right]+\operatorname{var}\left[\eta_{i}\right]}
$$

and this results in an error probability that is given by

$$
P_{e}[v]=\frac{1}{2}\left(1-\sqrt{\frac{\bar{\gamma}_{k}[v]}{1+\bar{\gamma}_{i}[v]}}\right)
$$

where $\bar{\gamma}_{i}[v] \triangleq \frac{1}{2} \mathrm{E}\left[S / N_{i}[v]\right]$ and again the expectation is with respect to $\beta$.

We conclude this section by highlighting an important point which makes our proposed analysis different from the one presented in [8]. As mentioned earlier, the proposed interference cancellation method reduces to the one presented in [8] when $\mathcal{T}_{v}^{(k)}=0$ for all $v$ and $k$ (i.e., when a hard decision is performed upon the correlator outputs). In this case, the $v$ th stage SNR for user $i$ and for a given $\beta=\beta_{1}^{(i)}$ is given by (26) with $\mathcal{T}_{v}^{(k)}=0$ for all $v$ and $k$ ). Since $\beta$ is a random quantity then the system error probability must be obtained by expressing the conditional error probability $P_{e}(\beta)$ as a function of this SNR and then integrating $P_{e}(\beta)$ with respect to the distribution of $\beta$. Instead of doing so, however, the analysis of [8] proceeds by defining the $v$ th stage SNR for user $i$ as

$$
\operatorname{SNR}_{i \text { Yoon }}(v)=\frac{\mathrm{E}\left[\beta^{2} \frac{P_{i}}{2} T^{2}\right]}{\operatorname{var}\left[\tilde{C}_{i}[v]\right]+\operatorname{var}\left[\tilde{S}_{i}[v]\right]+\operatorname{var}\left[\eta_{i}\right]}
$$

and then expressing the average error probability as

$$
P_{\text {YYoon }}[v]=Q\left[\sqrt{\operatorname{SNR}_{\text {YYon }}(v)}\right]
$$

In other words, the expectation with respect to the path amplitude $\beta$ is performed inside the $Q[\cdot]$ function. It turns out that the BER expression as defined in (29) is relatively accurate under ideal power conditions and when the path amplitudes are constants (see [14] for instance). This is further supported by the numerical results presented in [8] where the path amplitudes were all set to one. However, when the path amplitudes are random as in this paper then (29) will tend to significantly underestimate the system's BER's as shown in the numerical results section. Nevertheless, it is important to emphasize that the analysis of [8] is nice because of its simplicity.

\section{OPTIMIZATION}

\section{A. The Initial Stage Thresholds}

This section briefly describes a simple analytical approach for finding the optimal thresholds $\mathcal{T}_{0 \text {,opt }}^{(k)}$ for the $k$ th user $(k=1,2, \cdots, K)$ at the initial stage. From (22) and (23), it follows that we must minimize (18) and (19) in order to improve the detector performance. A close observation of (18) and (19) then indicates that the system performance can be optimized by minimizing $\mathrm{E}\left[\left(\widetilde{b}_{n}^{(k)}[0]\right)^{2}\right]$ as defined in (21) for all $k=1,2, \cdots, K$. In particular, the optimal value of $\xi_{0}^{(k)}, \xi_{0, \text { opt }}^{(k)}$ which minimizes $\mathrm{E}\left[\left(\widetilde{b}_{n}^{(k)}[0]\right)^{2}\right]$ can be easily found numerically. As a result, it follows that the optimal threshold for the $k$ th user at the initial stage is

$$
\mathcal{T}_{0, \mathrm{opt}}^{(k)}=\xi_{0, \mathrm{opt}}^{(k)} \sqrt{\operatorname{var}\left[\tilde{C}_{k}[1]\right]+\operatorname{var}\left[\tilde{S}_{k}[1]\right]+\operatorname{var}\left[\eta_{k}\right]}
$$




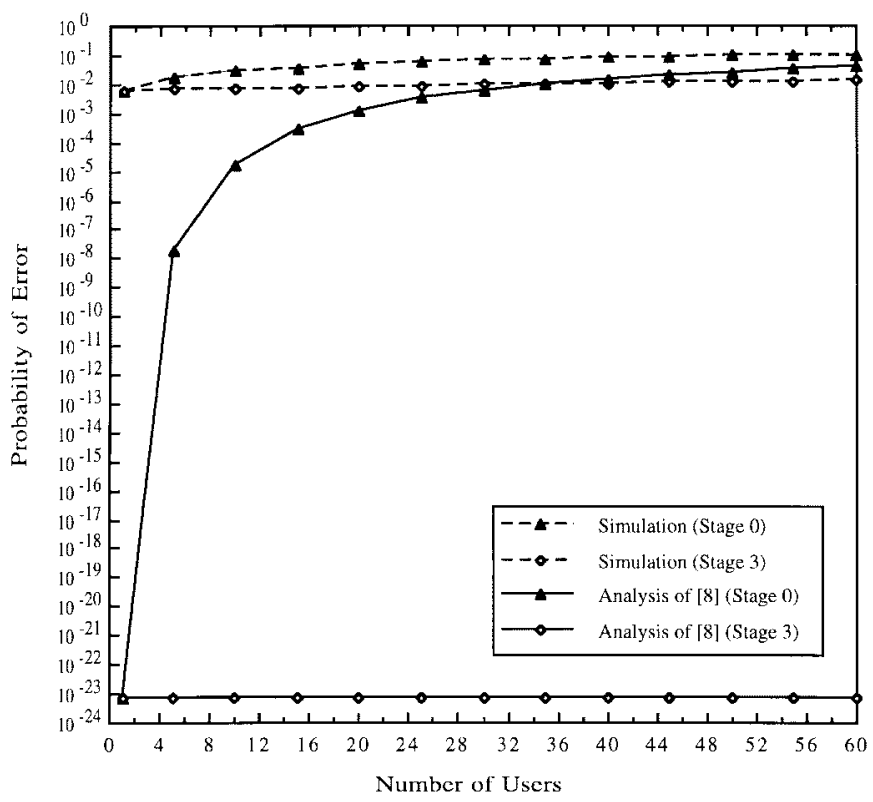

Fig. 2. A comparison between the Monte Carlo simulation BER estimates of the interference cancellation method of [8] with those obtained from the theoretical analysis of [8].

\section{B. The Successive Cancellation Stage Thresholds}

The extension of the approach described in the previous section to a $v$-stage detector is simple. Let $S / N_{k}[v]$ be defined as in (26), then $\mathrm{E}\left[\left(\tilde{b}_{n}^{(k)}[v-1]\right)^{2}\right]$ can be minimized for each user by finding the optimal value of $\xi_{v-1}^{(k)}, \xi_{v-1, \text { opt }}^{(k)}$, which minimizes $\mathrm{E}\left[\left(\tilde{b}_{n}^{(k)}[v-1]\right)^{2}\right]$ numerically. As a result, it follows that the optimal threshold for the $k$ th user is given by

$$
\mathcal{T}_{v-1, \mathrm{opt}}^{(k)}=\xi_{v-1, \mathrm{opt}}^{(k)} \sqrt{\operatorname{var}\left[\tilde{C}_{k}[v]\right]+\operatorname{var}\left[\widetilde{S}_{k}[v]\right]+\operatorname{var}\left[\eta_{k}\right]}
$$

Note that since we assume that the statistics of the channel characteristics are known, it follows that we would be able to set the value of the thresholds for all of the users before the data acquisition. Therefore, our proposed receiver will not cost much when compared to the one proposed in [8].

\section{NUMERICAL RESULTS}

This section presents some sample numerical results which illustrate the potential of using the proposed CI cancellation method as an effective tool for improving the performance of DS/CDMA communications over multipath fading channels. Throughout, we shall assume that the maximum delay spread $\Delta=7 \mu \mathrm{s}$ and that the data rate is equal to $9600 \mathrm{~b} / \mathrm{s}$. Unless otherwise mentioned, each user is assigned a unique balanced Gold sequence of length 127 that has been obtained from $m$-sequences that are represented by octal numbers 211 and 217 , respectively. Furthermore, the unit energy constraint (i.e., $\Sigma_{\ell=1}^{L_{i}} \operatorname{var}\left[\beta_{\ell}^{(i)}\right]=1$ ) will be assumed throughout.

We begin by investigating the accuracy of our analysis as well as the one presented in [8]. Figs. 2 and 3 present the average BER performance against the total number of users $K$ with $\left(\overline{E_{k} / N_{o}}\right) \triangleq \mathrm{E}\left[\left(\beta_{\ell}^{(k)}\right)^{2}\right]\left(E_{k} / N_{o}\right)=17 \mathrm{~dB}$ and $L_{k}=2$ for all $k=1,2, \cdots, K$. Specifically, Fig. 2 compares some sample BER results corresponding to the interference

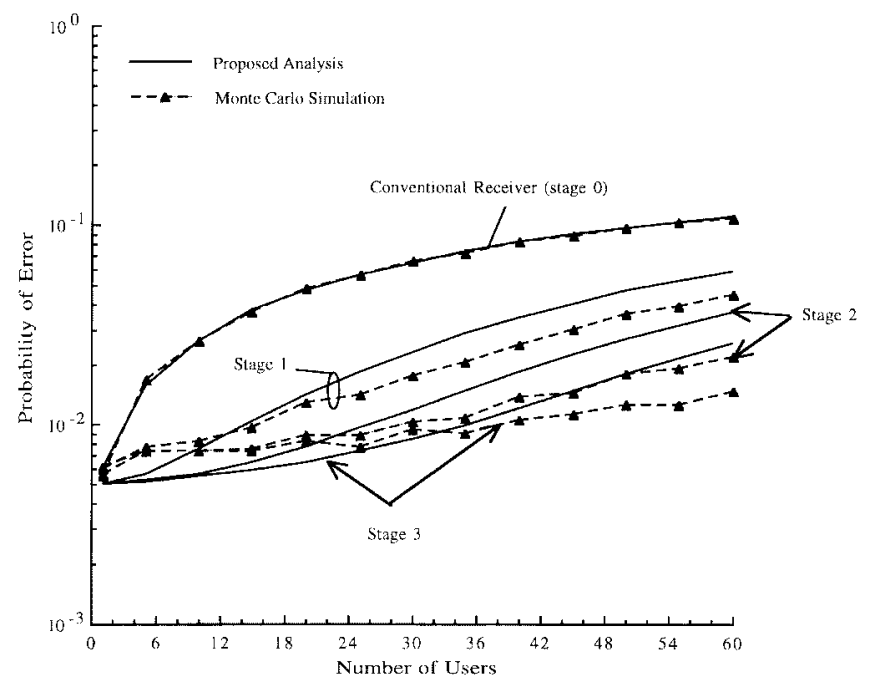

Fig. 3. An investigation of the accuracy of the proposed analysis.

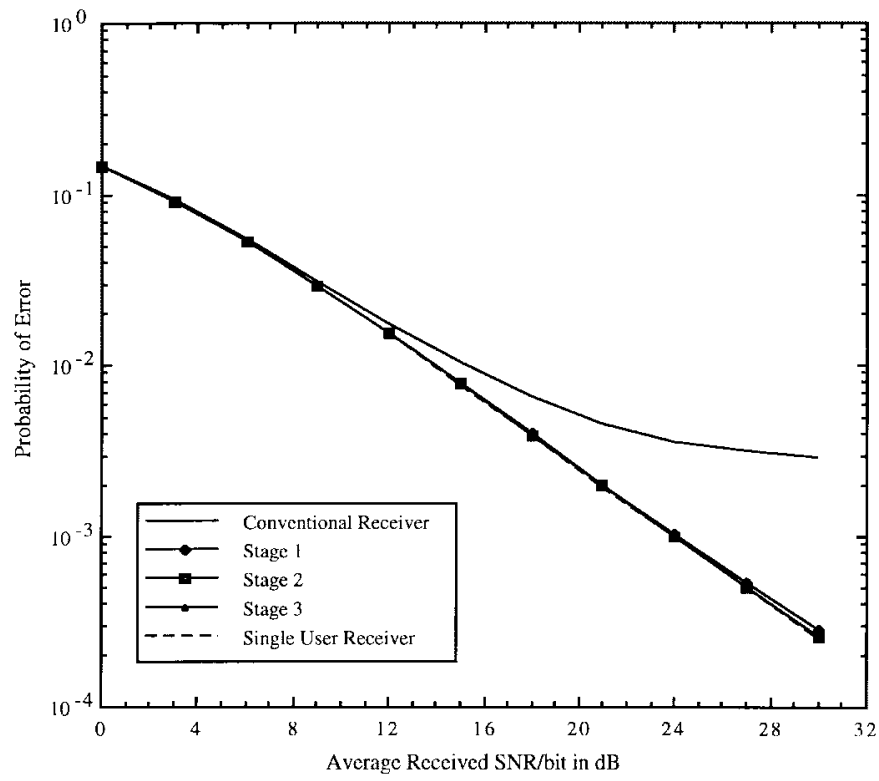

Fig. 4. The performance of the proposed interference cancellation method for a three-user DS/CDMA system.

cancellation method of [8] which were obtained by conventional Monte Carlo simulations with those obtained from the analysis of [8]. Likewise, Fig. 3 compares the BER estimates of our CI method which were obtained by Monte Carlo simulations with those based upon the analysis of Section IV. A close observation of both of these figures indicates that the error performance analysis of [8] tends to significantly underestimate the system's BER's, especially as the number of stages increases. In contrast, the analysis presented here gives BER estimates that are much closer to the true BER's as predicted by Monte Carlo simulations. These theoretical results are clearly not exact. However, because of the prohibitive amount of simulation time required to achieve accurate BER estimates, the proposal analysis could prove to be a useful tool for performance analysis and design of the proposed CI interference method as well as the one presented in [8].

Fig. 4 lists the performance of the proposed CI cancellation method against the average received SNR per bit $\bar{E}_{k} / N_{0}$ for 


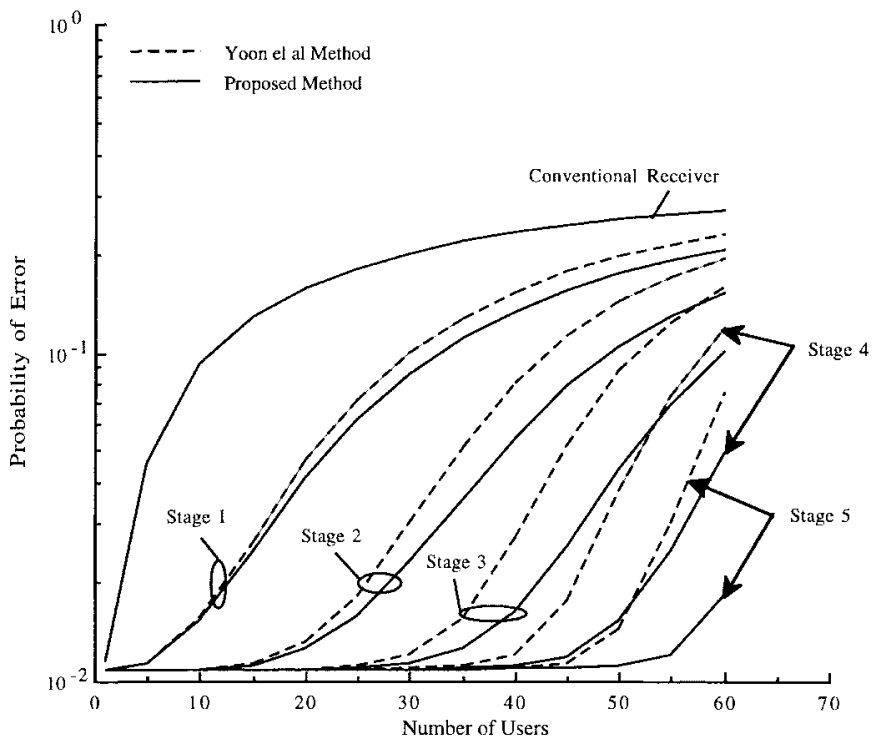

Fig. 5. The performance of the proposed interference cancellation method and the interference cancellation method of [8]. $E_{k} / E_{i}=4 \mathrm{~dB}$ for all $k \neq i$ and a Rayleigh multipath fading channel with $L_{k}=3$ are assumed.

a three-user system with $L_{k}=1$ for $k=1,2, \cdots, K$ and equal received power per user. A close observation of this figure clearly indicates the potential of the proposed method. In particular, it indicates that as the number of stages increases the system performance increases and that the system's BER as predicted by the proposed analysis will tend to approach the single user bit-error probabilities. Note that similar conclusions can be also drawn from Fig. 3 .

The remaining figures illustrate the potential of the proposed method in combating the effects of the near-far problem. Specifically, Fig. 5 lists the BER values of an asynchronous DS/CDMA employing Gold sequences of length 63 which have been obtained from $m$-sequences that are represented by octal numbers 103 and 147, respectively. Further, we assume that $L_{k} \equiv 3$ for all $k, E_{i} / N_{0}=9 \mathrm{~dB}, E_{k} / N_{0}=13$ $\mathrm{dB}$ for $k \neq i$, and constant path amplitudes for all data links. For comparison, we have also plotted the BER values corresponding to the detector proposed in [8]. Again note the BER performance improvement of the proposed detector as $V$ increases. Also note that the proposed detector provides near-far immunity even in multipath conditions and that it performs better than the one proposed in [8].

Fig. 6 lists the error probability of a DS/CDMA system employing Gold sequences with length 127 along with the proposed CI cancellation method as a function of the total number of users $K$ when $L_{k} \equiv 4$ and $\mathrm{E}\left[\left(\beta_{\ell}^{(k)}\right)^{2}\right]=0.25$ for $k=1,2, \cdots, K$. In particular, the received power of the desired user is $4 \mathrm{~dB}$ lower than the received power of all of the other users. For comparison, Fig. 6 presents in dashed lines the BER values obtained by the conventional receiver when $\overline{E_{k} / N_{o}}=30 \mathrm{~dB}$ for all $k$. Finally, Fig. 7 lists the Monte Carlo simulation estimates of the system's BER under the conditions of $L_{k} \equiv 4$ for all links, $\overline{E_{i} / N_{o}}=26$ $\mathrm{dB}$, and $\overline{E_{k} / N_{o}}=30 \mathrm{~dB}$ for $k \neq i$. For comparison, we have also plotted the simulation results corresponding to the detector proposed in [8]. Again note the BER performance

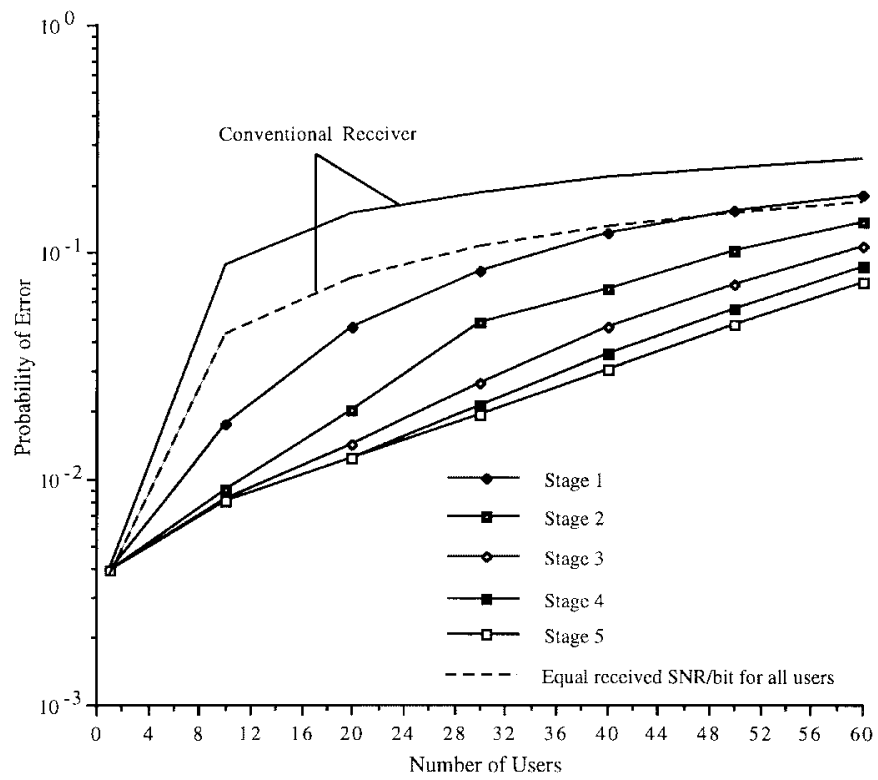

Fig. 6. The performance of the proposed interference cancellation method against the total number of users with and without equal average received SNR per bit of each user.

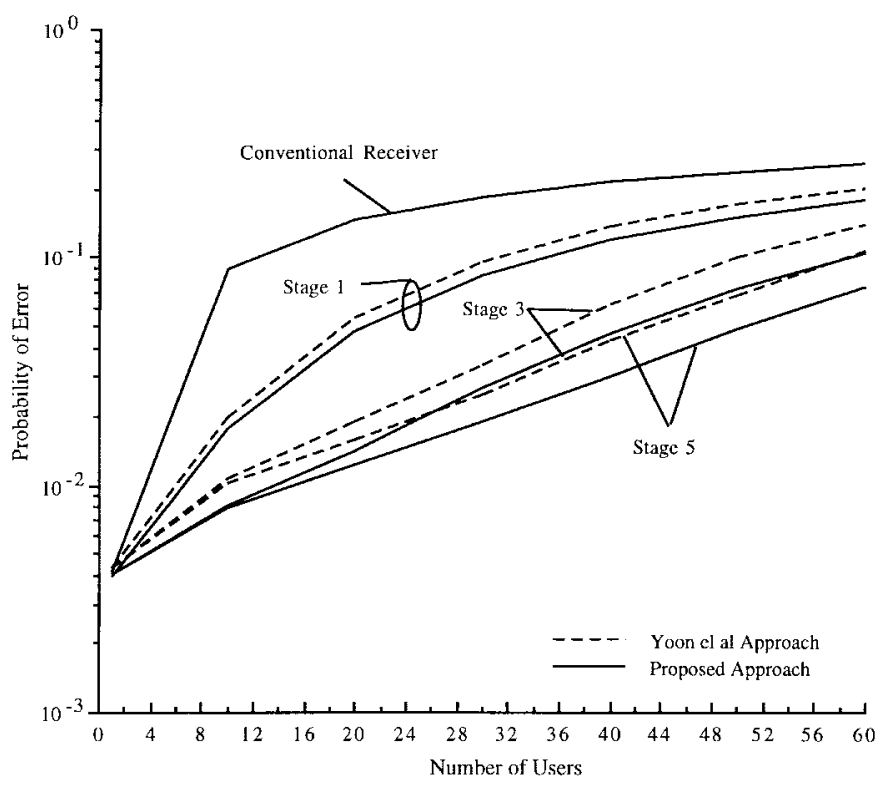

Fig. 7. The performance of the proposed interference cancellation method and the interference cancellation method of [8] with imperfect power control over a Rayleigh multipath fading channel with $L_{k}=4$.

improvement of the proposed detector. In particular, notice that the proposed detector performs better than the conventional receiver and, more importantly, it converges faster than the one proposed in [8]. For example, Fig. 7 shows that our three-stage CI cancellation detector is approximately the same as the five-stage CI cancellation detector proposed in [8], thereby implying a reduction in the hardware complexity and processing time compared to the CI cancellation method of [8].

\section{CONCLUSION}

CDMA is a spread-spectrum multiaccess technique which is currently the object of much attention. However, this tech- 
nique suffers from severe reductions in system performance because of the effects of near-far and CI. In this paper, we have proposed and analyzed a CI cancellation detector for demodulating asynchronous DS/CDMA signals over wireless channels that are characterized by multipath fading links. The proposed method, which is based on [8], is a successive CI rejection scheme which attempts to cancel the CI effects of the strongest signals from the received signal. Numerical results have demonstrated the effectiveness of the proposed method. In particular, it has been shown that the proposed analysis is consistent with Monte Carlo simulations. This is in contrast to the analysis presented in [8] which can significantly underestimate the true system's BER's as demonstrated in the previous section. It has been also demonstrated that the proposed cancellation strategy performs better than the one proposed in [8] without any additional complexity. Finally, it has been shown that the proposed cancellation strategy can alleviate the effects of the near-far problem and that significant system capacity improvement can be achieved using the proposed detector instead of the conventional DS/CDMA receiver.

\section{REFERENCES}

[1] K. B. Letaief, "The performance of optical fiber direct-sequence spreadspectrum multiple-access communications systems," IEEE Trans. Commun., vol. 43, pp. 2662-2666, Nov. 1995.

[2] R. Kohno, R. Meidan, and L. B. Milstein, "Spread spectrum access methods for wireless communications," IEEE Commun. Mag., vol. 33, pp. 58-67, Jan. 1995.

[3] S. Verdú, "Minimum probability of error for asynchronous Gaussian multiple-access channels," IEEE Trans. Inform. Theory, vol. IT-32, pp. 85-96, Jan. 1986.

[4] R. Lupas and S. Verdú, "Near-far resistance of multiuser detectors in asynchronous channels," IEEE Trans. Commun., vol. 38, pp. 496-508, Apr. 1990.

[5] M. K. Varanasi and B. Aazhang, "Multistage detection in asynchronous code-division multiple-access communications," IEEE Trans. Commun., vol. 38, pp. 509-519, Apr. 1990.
[6] X. Zhang and D. Brady, "Soft-decision muli-stage detection for asynchronous AWGN channels," in Proc. 31st Annu. Allerton Conf., UrbanaChampaign, IL, Oct. 1993, pp. 54-63.

[7] V. Vanghi and B. Vojcic, "Two-stage multiuser detection with soft interference cancellation," in Proc. CISS'95, Baltimore, MD, Mar. 1995, pp. 186-192.

[8] C. Y. Yoon, R. Kohno, and H. Imai, "A spread-spectrum multiaccess system with cochannel interference cancellation for multipath fading channels," IEEE J. Select. Areas Commun., vol. 11, pp. 1067-1075, Sept. 1993

[9] A. Duel-Hallen, "Decorrelating decision-feedback multiuser detector for synchronous code-division multiple-access channels," IEEE Trans. Commun., vol. 41, pp. 285-290, Feb. 1993.

[10] P. Patel and J. M. Holtzman, "Analysis of a simple successive interference cancellation scheme in a DS/CDMA systems," IEEE J. Select. Areas Commun., vol. 12, pp. 796-807, June 1994.

[11] G. L. Turin, "The effects of multipath and fading on the performance of direct-sequence CDMA systems," IEEE Trans. Veh. Technol., vol. VT-33, pp. 213-219, Aug. 1984.

[12] K. B. Letaief, "Efficient evaluation of the error rate probabilities of spread-spectrum multiple-access communications," IEEE Trans. Commun., vol. 45, pp. 239-246, Feb. 1997.

[13] M. B. Pursley, D. V. Sarwate, and W. Stark, "Error probability for direct sequence spread spectrum multiple-access communications-Part I: Upper and lower bounds," IEEE Trans. Commun., vol. COM-30, pp. 975-984, 1982.

[14] K. B. Letaief, K. Muhammad and J. S. Sadowsky, "Fast simulation of DS/CDMA with and without coding in multipath fading channels," IEEE J. Select. Areas Commun., vol. 15, pp. 626-639, May 1997.

Andrew L. C. Hui received the B.Eng. and the M.Phil. degrees in electronic engineering from the Hong Kong University of Science and Technology (HKUST), Clear Water Bay, Kowloon, Hong Kong, in 1994 and 1996, respectively.

From 1994 to 1996 he was with HKUST as a Research Assistant conducting research on DS/CDMA. His research interests include cochannel interference cancellation in DS/CDMA systems and frequency planning algorithms in GSM systems.

Khaled Ben Letaief (S'85-M'86-SM'97), for photograph and biography, see p. 285 of the February 1998 issue of this TRANSACTIONS. 